\title{
Ubiquitous Total Station Development using Smartphone, RSSI and Laser Sensor providing service to Ubi-GIS
}

\author{
M.H. SHoushtari ${ }^{\text {a, }}$, A. Sadeghi-Niaraki ${ }^{\text {a }}$ \\ ${ }^{a}$ GIS Dept., Geoinformation Technology Center of Excellence, Faculty of Geodesy \& Geomatics Engineering, K.N.Toosi University \\ of Technology, Tehran, Iran \\ shushtari.m.h@gmail.com, a.sadeghi@kntu.ac.ir
}

KEY WORDS: Ubiquitous Total Station, Triangle method, RSSI, Laser sensor, Smartphone, Ubiquitous surveying

\begin{abstract}
:
The growing trend in technological advances and Micro Electro Mechanical Systems (MEMS) has targeted for intelligent human lives. Accordingly, Ubiquitous Computing Approach was proposed by Mark Weiser. This paper proposes an ubiquitous surveying solution in Geometrics and surveying field. Ubiquitous Surveying provides cost-effective, smart and available surveying techniques while traditional surveying equipment are so expensive and have small availability specially in indoor and daily surveying jobs. In order to have a smart surveying instrument, different information technology methods and tools like Triangle method, Received Signal Strength Indicator (RSSI) method and laser sensor are used. These new ways in combine with surveying equations introduces a modern surveying equipment called Ubi-Total Station that also employed different sensors embedded in smartphone and mobile stand. RSSIbased localization and Triangle method technique are easy and well known methods to predict the position of an unknown node in indoor environments whereas additional measures are required for a sufficient accuracy.
\end{abstract}

In this paper the main goal is to introduce the Ubiquitous Total Station as a development in smart and ubiquitous GIS. In order to public use of the surveying equipment, design and implementation of this instrument has been done. Conceptual model of Smartphonebased system is designed for this study and based on this model, an Android application as a first sample is developed. Finally the evaluations shows that absolute errors in X and $\mathrm{Y}$ calculation are 0.028 and 0.057 meter respectively. Also RMSE of 0.26 was calculated in RSSI method for distance measurement. The high price of traditional equipment and their requirement for professional surveyors has given way to intelligent surveying. In the suggested system, smartphones can be used as tools for positioning and coordinating geometric information of objects.

\section{INTRODUCTION}

Technological development dramatically has made changes in various aspects of human life since last few years. In this way, development of communication technology has held an important role. In ubiquitous computing approach (Weiser, 1991), people can satisfy their needs in any context, in any location, at any time and by any user; using embedded sensor (Sadeghi-Niaraki, 2013).

Geomatic sciences has been affected by new information technology just like the other ones. Due to increasing public access to smartphones, Volunteered geographic information (VGI) and Crowdsourcing is growing significantly (Ogundipe, 2013), In other words, we are among the viewers of numerous applications of IT and geomatics in social life of people. In some researches GPS is introduced as the only ubiquitous localization system available, it generally provides only absolute coordinates (Hedgecock et al, 2013); Thus, ubiquitous surveying tools are known as compatible, available, public and new generation of surveying equipment (Shoushtari et al, 2013)

Ogundipe (2013) seed the smartphone surveying tools with emphasis on the GPS sensor. Surveying equations are used in combine with smartphone data calculation coordinates and error vectors. Also GPS sensor of Smartphone was employed as a webGIS data collector by Lwin and Murayama (2011).

Although surveying equipment did not developed for public use but it is evident in human life; take measuring length, area and a few more. as examples. Also surveying specialist don't need very high accuracy surveying in many jobs. Daily check-up and some part of road and structure projects just need quick and available tools to prove the job accuracy. So the lack of public use's potential, high price, high weight and high volume of traditional surveying equipment are limited issues and problems that have been solved by using information technology tools and methods like smartphone and RSSI technique.

The RSSI-based localization technique is an easy and well known method to predict the position of an unknown point in indoor environments whereas additional measures are required for a sufficient accuracy. The distance between smartphone and Wi-Fi detector estimated by received signal strength and least square estimation (LSE) is used to calculate the position of the unknown point (Wang et al., 2010).

Researchers used the other information technology tools to determinate three-dimensional location. Jimenez et al. (2009) believed the traditional measurement tools like Total Station, photogrammetry and DGPS are more effective but have lots of limitations. In this research the typical accuracy is achieved 10$\mathrm{mm}$ in nature condition using acoustic signals which provide sketching objects with enough detail. It can be seen that there is no need for accuracy of $1 \mathrm{~mm}$ in some projects, also the effect of new method on surveying is shown.

In this paper the main goal is to introduce of Ubi-Total Station using smartphone and RSSI techniques. A simple tool as the first generation of Ubi-Total Station that use smartphone sensors to measure coordinate was created. In this research, three specific ways by Total Station using smartphone will be shown and finally an electronic circuit will be proposed. 


\section{METHODOLOGY}

Ubi-Total Station design procedure using smartphones with built-in cameras, sensors and etc. will be described in this section. First, traditional Total Station components was studied. Next, according to this study; the main components were modeled.

\subsection{GPS-Total Station Components}

At the heart of the GPS Total Station is a small, highly-integrated GPS receiver and radio modem. In GPS Total Station system it is a truly integrated all elements: radios, survey controller, and office software. The GPS Total Station is quite simply the most advanced and versatile survey instrument have ever built (Total Station.org, see 2013). It can be named the main sample of UbiTotal Station that has this four Components (Jazireeyan, 2008):

1. Centre of GPS data receive

2. Centre of distance measurement

3. Centre of angle measurement

4. Calculation centre

\subsection{Ubi-Total Station modelling}

Ubi-Total Station introduced in this study can be divided into four components, just like the traditional one. GPS sensor, distance measurement tools and techniques, smartphone sensors like gyroscope and camera sensor, specific method for calculation were employed to provide the first generation of UbiTotal Station (see Figure 1).

2.2.1 Ubi-Total Station's Centre of GPS data receive: Only the top-level Total Stations have GPS receiver, so it is necessary for user to use a handheld GPS to know his approximate position and also to find a benchmark point. Ubi-Total Station can provide all handheld GPS's facilities using GPS sensor embedded in smartphone and also can get an approximate point to start surveying in outdoor environments.

2.2.2 Ubi-Total Station's Centre distance measurement: This Centre is the most important component in Ubi-Total Station. Because of lack of a sensor that would measure distance between smartphone and the target point, it is needful to find some way to solve this problem. These three method are offered in this study:
1. Triangle method
2. RSSI method
3. Laser sensor

Triangle method is used when the Ubi-Total Station user and the target point stand in the equal height (Figure 2). $\mathbf{\Delta} A A^{\prime} B$ is a solvable triangle by determining the $\Theta$ using gyroscope sensor and known height named $\mathrm{h}$. So the distance between smartphone $\left(A^{\prime}\right)$ and the target point (B) can be calculated (Shoushtari et al., 2013)

$$
A^{\prime} B=\frac{h}{\sin (90-\theta)}
$$

Where

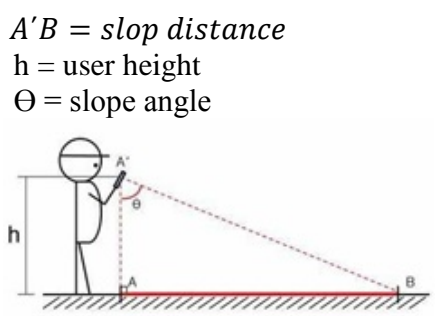

Figure 2. Triangle method: $A A^{\prime} B$ is the target triangle.

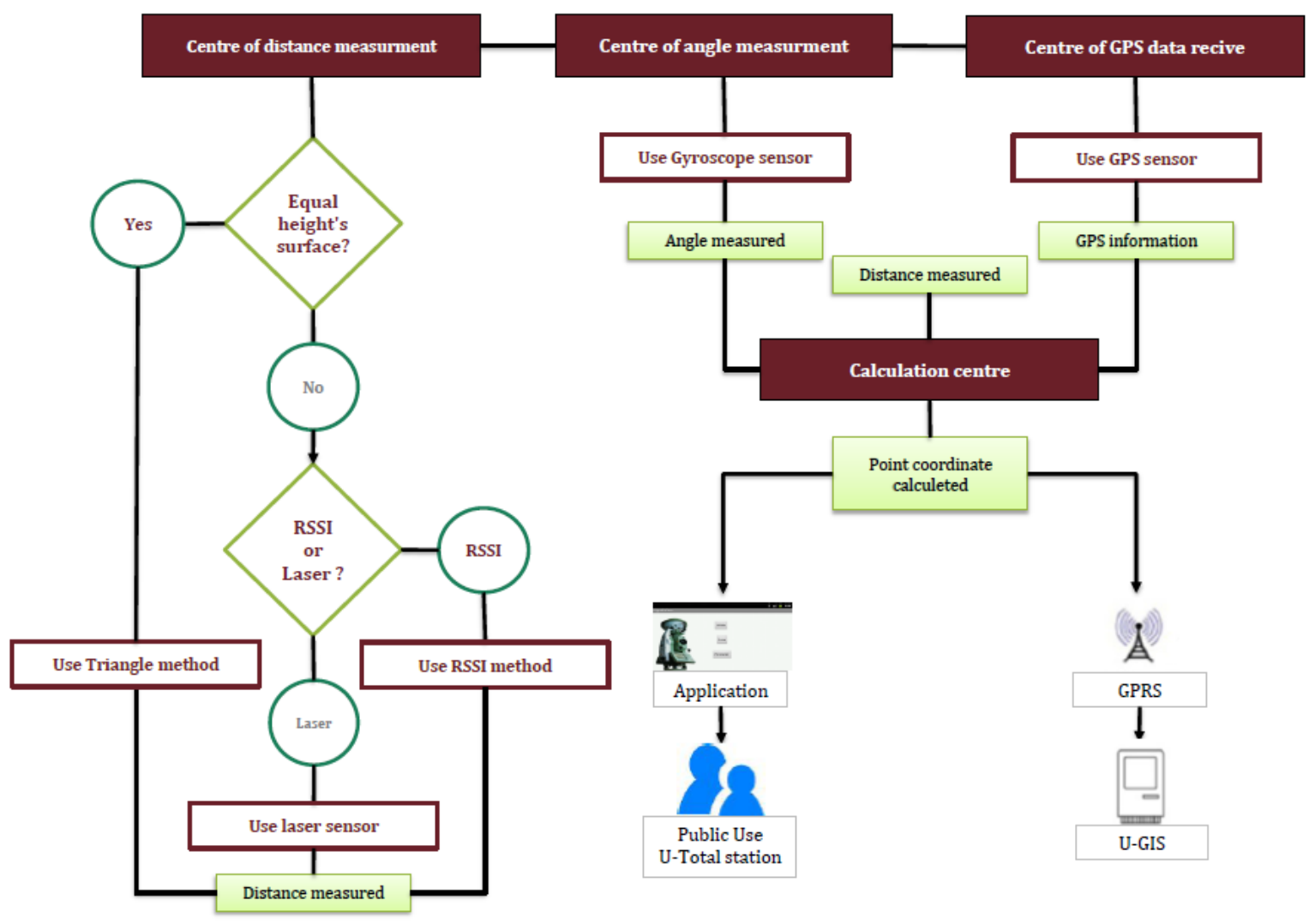

Figure1. Conceptual diagram of Smartphone-based system designed for this study: the Ubi-Total Station application developed for this study using an Android 3.2 SDK. 
RSSI method is employed to measure the goal distance. The distance between smartphone and the receiver can be estimated by RSS, TOA (Time of Arrival), received signal phases and other techniques (Wang et al., 2010). In this research, the distance is produced by the measurement value of RSS according to an algorithm describing the relation estimation between receiver and sender.

Laser sensor is the simplest way to measure the goal distance. In this way, an electronic circuit measure the slope distance using a laser sensor. The measured data is sent to smartphone by sending SMS or email using GPRS. Conceptual diagram of UbiTotal Station stand included the electronic circuit is shown in figure 4 .

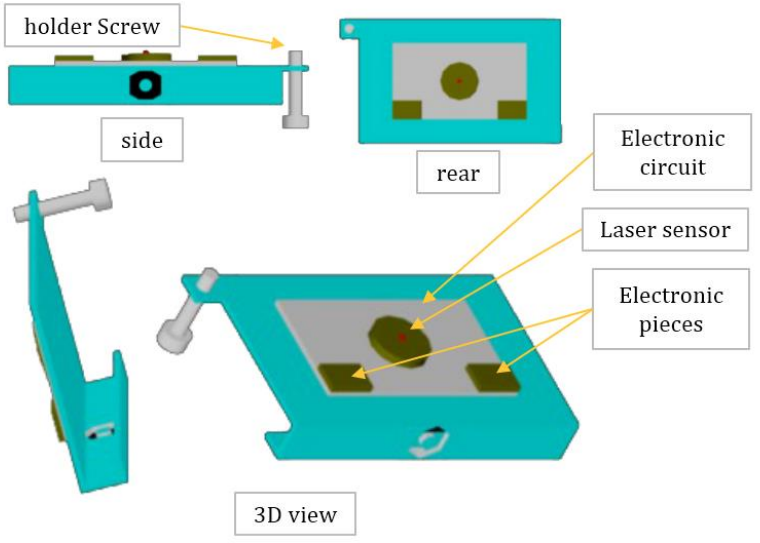

Figure 3. Conceptual diagram of Ubi-Total Station stand

2.2.3 Ubi-Total Station's Centre of angle measurement: Orientation is define as a combination of three angular quantities: Azimuth, pitch, and roll (Figure 5). Horizontal and vertical angles are calculated by measures. Gyroscope sensor embedded in smartphones provide all the angles that are needed.

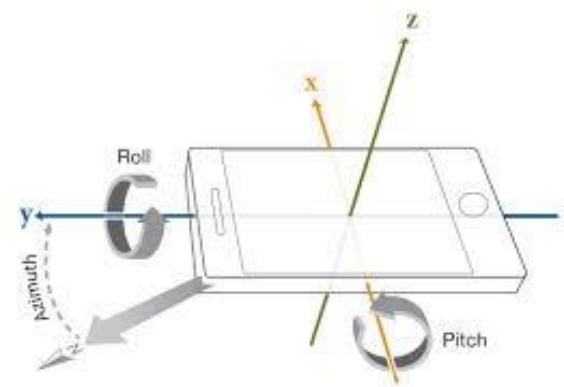

Figure 4. Azimuth, pitch, and roll in smartphones (matchwork.com, seen 2014)

2.2.4 Ubi-Total Station's calculation Centre: In a nutshell, a smartphone is a device that lets us to make telephone calls, but also adds in features that, in the past, it would has been found only on a personal digital assistant or a computer. Such as the ability to send and receive e-mail and edit Office documents, for example. Complex calculations of Ubi-Total Station are done by programing in smartphones. Android platform and IOS operating system are the most powerful tools for programing and calculation, so Ubi-GIS can develop in smartphones easily.

\section{IMPLEMENTATION}

In this paper, implementation of the Smartphone-based system designed using RSSI technique was set as the main goal. For this study an android 3.2 SDK (Gingerbread) was used to develop the android application. According to observations Eclipse IDE JUNO (Integrated development environment); a tool for Java developer creating Java EE and Android application; was selected, it is known as an available, user friend and well known IDE in programmer society. Figure 6 is shown the implemented application that include slope distance, direct distance, horizontal and vertical angle and a simple calibration named percentage calibration. Finally local 3D coordinate of target point will calculate. In order to obtain more realistic values of the results, the percentage calibration system has been designed. When a user have a known length in its measurement range, this will force as a fixed length to make estimation more acceptable. Several capabilities can be considered for the system, measurement and calculation of width, area and Perimeter was implemented as the sample of public use application.

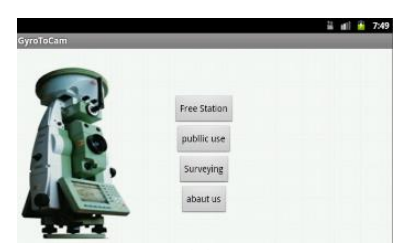

(a)

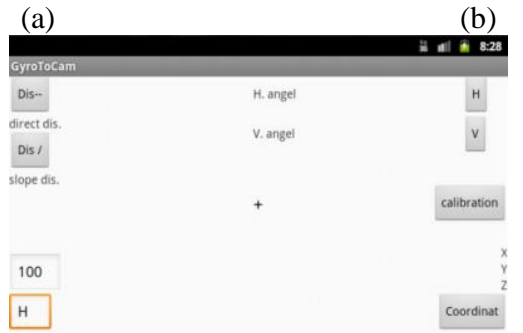

(c)

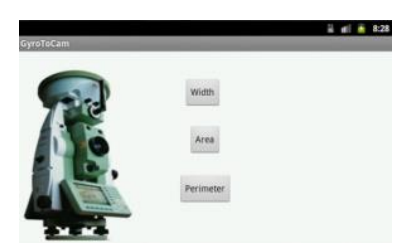

(b)

(c) application:

(a) first page, (b) public use page, (c) surveying page

Free station page will be the centre of settings. User can enter a local position for smartphone or use GPS sensor. Also three method of distance measurement will be available and finally user should use benchmark point to set the fixed coordinate system. In this android application, a local coordinate was entered as the station point and triangle method was set as the default method for distance measurement. RSSI method and laser sensor implementation for distance measurement will be examined separately.

In RSSI method, In order to estimate the location of receiver in two dimensional space, at least three measurements will be needed. Figure 6 shows the three measurement of positioning. Thus, user have to provide three benchmark points just for $2 \mathrm{D}$ result.

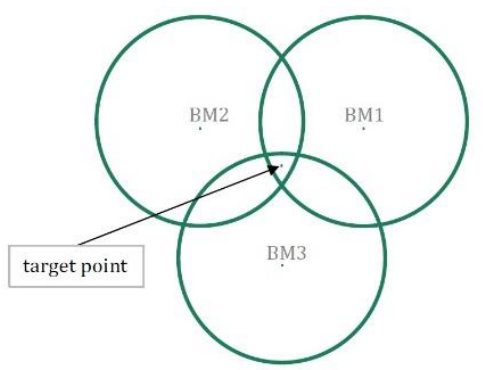

Figure 6. Three measurement of positioning in RSSI method 
If the distance estimation value between receiver and smartphone is $d$, receiver can be positioned to a circle and the smartphone as the centred, $d$ as the radius of the circle. The second measure reduces the ambiguity of positioning, and the target point positioned in two crossing circular arc. The third measure locks the position of receiver.

In order to present how this method works, the goal distance called d (distance between smartphone and receiver) was define as a function of RSSI values. In general, the least square method can be used for the processing of linear parameters, can also be used for the processing of nonlinear parameters Wang et al. (2010) considered global polynomial to make the measurement of smartphone Bluetooth RSSI. So relation 2 was established as the main relation for LSE calculation.

$$
d=a_{1}+a_{2} x+a_{3} x^{2}
$$

Where

$$
\begin{aligned}
& d=\text { Known distance } \\
& a_{i}=\text { Unknown parameters } \\
& x=\text { Value of } R S S I
\end{aligned}
$$

Seven known length were considered, five distances as the control length to determine the parameters and two distances as check length. Table (1) shows the measurement and known distances.

\begin{tabular}{|c|c|c|c|}
\hline Distance (d) & RSSI (x) & $4 \mathrm{~m}$ & $69 \mathrm{dBm}$ \\
\hline $1 \mathrm{~m}$ & $48 \mathrm{dBm}$ & $6 \mathrm{~m}$ & $77 \mathrm{dBm}$ \\
\hline $2 \mathrm{~m}$ & $55 \mathrm{dBm}$ & $8 \mathrm{~m}$ & $82 \mathrm{dBm}$ \\
\hline $3 \mathrm{~m}$ & $60 \mathrm{dBm}$ & $9 \mathrm{~m}$ & $85 \mathrm{dBm}$ \\
\hline
\end{tabular}

Table 1. Known distance and symmetric RSS for seven lengths

A smartphone that turn on its Wi-Fi hotspot as the sender and another smartphone that install a RSSI reader application (called Wifi Info II) was established to do these measurements. Suppose that the number of equation is five, then we have:

$$
A=\left[\begin{array}{rrr}
1 & x_{1} & x^{2} \\
\vdots & \vdots & \vdots \\
1 & x_{5} & x^{2}
\end{array}\right] \quad L=\left[\begin{array}{c}
d_{1} \\
d_{2} \\
\vdots \\
d_{5}
\end{array}\right\rfloor \quad X=\left\lfloor\begin{array}{l}
a_{1} \\
a_{2} \\
a_{3}
\end{array}\right\rfloor
$$

Where:

$$
\begin{aligned}
& A=\text { Coefficients matrix } \\
& L=\text { Observation matrix } \\
& X=\text { Unknowns matrix }
\end{aligned}
$$

Therefore, the main parameters that can be obtained by the following formula:

$$
X=\left(A^{T} A\right)^{-1} A^{T} L
$$

Table (2) shows the result of calculation:

\begin{tabular}{|c|c|c|}
\hline$a_{1}$ & $a_{2}$ & $a_{3}$ \\
\hline 8.18 & -0.35 & 00.00 \\
\hline
\end{tabular}

In order to obtain acceptable result and equal situation in each station, a simple stand showed in figure 7 was used. Also it is used for Smartphone-based system.

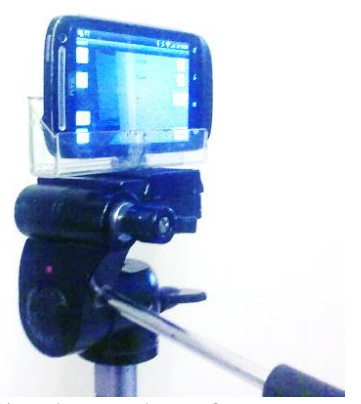

Figure 7. Simple stand use for measurements

Laser sensor is the third method that is used for distance measurement. An electronic circuit was designed to attach to the smartphone stand. Figure 8 shows the laser sender 5 mill watts and receiver sensor. The electronic circuit established in smartphone stand (designed in figure 3) will present in future research.

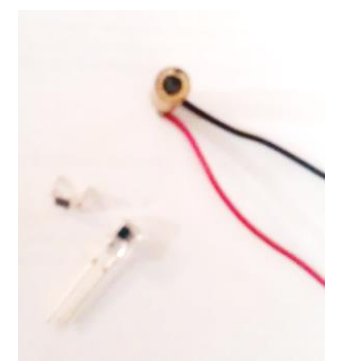

Figure 8. Laser sender and receiver sensors.

\section{EVALUATION}

Smartphone-based system finally was evaluated in an indoor environment. This test was done by a Trimble Total Station, also the results without any fixed length and percentage calibration are shown in table (3). These result was gained by the android application based on triangle method. In order to have more accurate measurement, user can use laser sensor attaining distance errors below $10 \mathrm{~mm}$.

\begin{tabular}{|c|c|c|c|c|}
\hline & $\begin{array}{c}\text { Slop dis. } \\
(\mathrm{mm})\end{array}$ & $\begin{array}{c}\text { Direct dis. } \\
(\mathrm{mm})\end{array}$ & $\begin{array}{c}\mathrm{X} \\
(\mathrm{mm})\end{array}$ & $\begin{array}{c}\mathrm{Y} \\
(\mathrm{mm})\end{array}$ \\
\hline Trimble & 1722 & 1142 & 31.347 & 40.103 \\
\hline System & 1711 & 1079 & 31.319 & 40.046 \\
\hline Error & 21 & 63 & 0.028 & 0.057 \\
\hline
\end{tabular}

Table 3. The result and evaluation of designed system

According to questionnaires that filled by surveyor, more than 90 percentage of surveying Specialist in statistic society prefer to use smartphone instead of big, heavy and expensive equipment.

In order to evaluate RSSI method for distance measurement, check points and RMSE (Root-Mean-Square Error) calculation showed in relation 5 were used.

Where

$$
R M S E=\sqrt{\frac{\sum\left(d_{\text {computed }}-d_{\text {observered }}\right)^{2}}{C L-1}}
$$

$d_{\text {computed }}=$ computed length by relation 2

$d_{\text {observered }}=$ observered length as the check length

$C L=$ number of known length as the control length

So it is calculated:

$$
R M S E=0.26
$$


The result shows RSSI technique is the operational one that can be used for distance measurements. It should admit that accurate RSSI is used by the other android applications, also more RSSI measurement and calibration can improve the RMSE.

\section{CONCLUSION}

In this study, capabilities of smartphone sensors such as orientation and camera sensors were used for professional user and public use. Based on ubiquitous surveying approach, embedded sensors such as laser sensor stablished on smartphone stand was offered. With this approach, Ubi-Total Station as a popular surveying equipment was implemented. The acceptable evaluations has verified the feasibility of ubiquitous surveying tools using information technology and new methods.

The result gained in RSSI method showed that this way can be a suitable replace for reflector-based Total Station. There are a few way that can improve the results like positioning LSE calculation, ubiquitous levelling technique and RSSI method in combine with centre of angle measurement.

Although GPS sensor was used before as a data collector in GIS and its services, but the new generation of ubiquitous surveying called Ubi-Total Station can develop the use's state of new generation of GIS named Ubi-GIS in indoor and outdoor environments.

Generally, IT will create drastic changes in geomatics science. Ubiquitous Surveying provides cost-effective, smart and available surveying techniques. There are various sensors in smartphones today that can be used for measuring the environment and positioning. Hence, proper utilization of these sensors marks many innovative and functional applications of this technology. That is why many researchers in the field have concentrated on using IT methods and smartphones as the devices for design and implementation of modern surveying equipment. It seems, surveying specialist will use some modern, small, lightweight and accurate equipment in future.

In the future research, a new android application that employ three distance measurement methods will be introduced. Smartphone-based system with laser sensor established in stand is going to be a patent.

\section{REFERENCES}

Gervais, E.; Liu, H.; Nussbaum, D.; Roh, Y.S.; Sack, J.; Yi, J. 2007 "Intelligent map agents-An ubiquitous personalized GIS, School of Computer Science", Carleton University, 1125 Colonel By Drive, Ottawa, Canada K1S 5B6

Goodchild, M.F.; 2007, "Citizens as sensors: the world of volunteered geography"; Geo Journal 69 (4): 211-221

Heipke, C.; 2010, "Crowdsourcing Geospatial Data". ISPRS Journal of Photogrammetry and Remote Sensing 65, pp. 550 557.

Jazireeyan, I; 2008,"Advanced Surveying Instruments", Book ID: $978-064-2898-04-9$
Jiménez, A.R; Prieto, J.C; Ealo, J.L; Guevara, J; Seco, F; 2009, "A computerized system to determine the provenance of finds in archaeological sites using acoustic signals", Journal of archaeological science, volume 36, Issue 10, pages 2415-2426

Keller, F.; Willemsen, T; Sternberg, H; "Calibration of Smartphones for the use in indoor navigation", 978-1-46731954-6/12/\$31.00 @2012 IEEE

Longley, P.A. 2001, Goodchild, M.F.; Maguire, D.J.; Rhind, D.W. "Geographic Information Systems and Science". John Wiley \& Sons.

Ogundipe, O.; 2013, "The Smartphone as a Surveying Tool”, TS03C - Positioning and Navigation - Today and Tomorrow 6626

Sadeghi-niaraki, A; 2013, "Ubiquitous GIS, new generation of GIS", SMPR international conference, university of Tehran

Shoushtari, M.h; Habibi, R; Sadeghi-Niaraki, A; fall 2013, "Introducing a ubiquitous surveying equipment", K.N.Toosi university exhibition as a research plan

Shoushtari, M.h; Habibi, R; Sadeghi-Niaraki, A; February 2013, "Design and implementation of ubiquitous surveying equipment", $32^{\text {nd }}$ national \& $1^{\text {st }}$ International geoscience congress

Wang, Y.; Shi, SH.; Yang, X.; Ma, A.; 2010 "Bluetooth indoor positioning using RSSI and least square estimation"

Hedgecock, W.; Maroti, M.; Sallai, J.; Volgyesi, P.; Ledeczi, A.; 2013, "High-accuracy differential tracking of low-cost GPS receivers" 11th annual international conference on Mobile systems application and services, pages 221-234

Weiser, M. (1991). The computer for the 21st century. Scientific American, 265(3), 94-104.

http://mobiledeviceinsight.com/2011/12/sensors-in-smartphones http://totalstation.org/all-about-gps-total-station.php 